\title{
Novel genomic findings in multiple myeloma identified through routine diagnostic sequencing
}

\author{
Georgina L Ryland, ' Kate Jones, ${ }^{1}$ Melody Chin, ${ }^{2}$ John Markham, ${ }^{3}$ Elle Aydogan, ${ }^{1}$ \\ Yamuna Kankanige, ${ }^{1}$ Marisa Caruso, ${ }^{4}$ Jerick Guinto, ${ }^{1}$ Michael Dickinson, ${ }^{4,5}$ \\ H Miles Prince, ${ }^{4,5}$ Kwee Yong, $^{2}$ Piers Blombery ${ }^{1,5}$
}

\begin{abstract}
- Additional material is published online only. To view please visit the journal online (http://dx.doi.org/10.1136/ jclinpath-2018-205195).

${ }^{1}$ Department of Pathology, Peter MacCallum Cancer Centre, Melbourne, Victoria, Australia ${ }^{2}$ Department of Haematology, University College London Cancer Institute, London, UK ${ }^{3}$ Research Division, Peter MacCallum Cancer Centre, Melbourne, Victoria, Australia ${ }^{4}$ Department of Haematology, Peter MacCallum Cancer Centre, Melbourne, Victoria, Australia ${ }^{5}$ Sir Peter MacCallum Department of Oncology, University of Melbourne, Melbourne, Victoria, Australia
\end{abstract}

\section{Correspondence to}

Dr Piers Blombery, Department of Pathology, Peter MacCallum Cancer Centre, 305 Grattan St, Melbourne, Victoria, 3000, Australia; piers.blombery@ petermac.org

Received 4 April 2018 Revised 20 April 2018 Accepted 20 April 2018 Published Online First 14 May 2018
Check for updates

To cite: Ryland GL, Jones $\mathrm{K}$, Chin $\mathrm{M}$, et al. J Clin Pathol 2018;71:895-899.

\section{ABSTRACT}

Aims Multiple myeloma is a genomically complex haematological malignancy with many genomic alterations recognised as important in diagnosis, prognosis and therapeutic decision making. Here, we provide a summary of genomic findings identified through routine diagnostic next-generation sequencing at our centre.

Methods A cohort of 86 patients with multiple myeloma underwent diagnostic sequencing using a custom hybridisation-based panel targeting 104 genes. Sequence variants, genome-wide copy number changes and structural rearrangements were detected using an inhouse-developed bioinformatics pipeline.

Results At least one mutation was found in $69(80 \%)$ patients. Frequently mutated genes included TP53 (36\%), KRAS (22.1\%), NRAS (15.1\%), FAM46C/DIS3 (8.1\%) and TET2/FGFR3 (5.8\%), including multiple mutations not previously described in myeloma. Importantly we observed TP53 mutations in the absence of a 17p deletion in $8 \%$ of the cohort, highlighting the need for sequencing-based assessment in addition to cytogenetics to identify these high-risk patients. Multiple novel copy number changes and immunoglobulin heavy chain translocations are also discussed.

Conclusions Our results demonstrate that many clinically relevant genomic findings remain in multiple myeloma which have not yet been identified through large-scale sequencing efforts, and provide important mechanistic insights into plasma cell pathobiology.

\section{INTRODUCTION}

Through large whole genome, whole exome and targeted sequencing efforts, multiple myeloma has become one of the most genomically well-characterised haematological malignancies. ${ }^{1-4}$ In addition to published cohorts, important resources such as data from approximately 1000 patients with myeloma that have undergone whole exome and whole transcriptome sequencing are also publicly available as part of the Multiple Myeloma Research Foundation Personalized Medicine Initiative CoMMpass study (https://research.themmrf.org and www.themmrf. org). The molecular data to date demonstrate that myeloma is a temporally and spatially heterogeneous malignancy characterised by early-onset chromosomal aneuploidies and translocations involving the immunoglobulin loci, followed by the acquisition of driver mutations in the RAS/MAPK pathway, MYC dysregulation (through translocation with multiple partners) and acquisition of TP53 mutation and copy number loss.

Despite the extensive myeloma genomic data sets available, novel genomic lesions continue to be discovered with potential further insights into the biology of this disease. ${ }^{5}$ The increase in routine genomic analysis in the diagnostic laboratory in patients with myeloma provides another potential source of novel genomic findings. We aimed to review data from myeloma patient samples referred to our diagnostic service (Peter MacCallum Cancer Centre, Molecular Haematology Laboratory) that have been sequenced using a hybridisation-based next-generation sequencing panel that detects sequence variants, genome-wide copy number changes and immunoglobulin translocations (the Peter MacCallum Cancer Centre PanHaem panel ${ }^{6}$ ) with a focus on novel genomic findings.

\section{MATERIALS AND METHODS}

\section{Patient samples and DNA extraction}

Samples received for diagnostic testing by the Molecular Haematology Laboratory (Peter MacCallum Cancer Centre) from patients with myeloma were included in this study (online supplementary table 1). This includes a cohort of patients with deletion of $17 \mathrm{p}$, the outcomes of which have been previously described. ${ }^{7}$ DNA was extracted from bone marrow aspirate samples with at least 30\% plasma cells (32 by morphological assessment and 54 enriched by immunomagnetic selection using CD138 + beadspost mononuclear cell isolation by density centrifugation) using DNeasy Blood and Tissue Kit reagents (Qiagen, Hilden, Germany).

\section{Mutation screening}

We performed targeted next-generation sequencing of 104 genes (listed in online supplementary table 2) selected from previous myeloma sequencing studies $^{24}$ or known to be relevant in other haematological malignancies. The custom SureSelect hybridisation-based capture panel (Agilent, California, USA) also included the immunoglobulin heavy chain (IGH) locus, including the entire constant region and covering the switch and enhancer regions, and $\mathrm{V}, \mathrm{D}$ and J segments as described previously. ${ }^{8}$ DNA (200-300 ng) was sheared by focused acoustic sonication (Covaris, Massachusetts, USA) and fragment libraries prepared using the KAPA Hyper Prep Kit according to standard protocols (Kapa Biosystems, Massachusetts, USA). Hybridisation capture was performed according to the Agilent SureSelectXT 
protocol, followed by sequencing of indexed libraries on an Illumina NextSeq (paired-end $75 \mathrm{bp}$ reads). After de-multiplexing and base calling, BWA-MEM ${ }^{9}$ was used to align reads to the human genome (GRCh37 assembly), followed by local indel realignment (using the GATK software ${ }^{10}$ ) and marking of duplicate alignments (using Picard; http://broadinstitute.github. io/picard/). Captured regions were sequenced to a mean depth of $700 \times$, which was sufficient data for $98 \%$ of target regions to achieve at least 100 -fold coverage.

Variants were called using Haplotype caller ${ }^{10}$ and annotated using Ensembl Variant Effect Predictor (V.78). Known polymorphisms and common sequencing artefacts were excluded by filtering for minor allele frequencies $<0.05 \%$ (in the 1000 Genomes, ExAC, gnomAD or Exome Variant Server databases) and by manual review in Integrative Genomics Viewer (IGV). Variants overlapping coding regions and canonical splice sites were curated for pathogenicity, including previous description in the literature and cancer databases (eg, the Catalogue of Somatic Mutations in Cancer (COSMIC)) and in silico prediction. Only variants considered pathogenic are reported and are listed in online supplementary table 3 .

\section{Copy number analysis and translocation detection}

Copy number variants were estimated by comparing read counts from on-target and off-target reads with a pooled reference to correct for enrichment and sequencing biases (J Markham, 2018, manuscript in preparation). All samples were assessed for common copy number aberrations in myeloma (loss of $1 \mathrm{p}$, gain of 1q, monosomy 13, loss of $17 \mathrm{p}$ ), as well as novel changes. The Genomic Rearrangement IDentification Software Suite (GRIDSS) was used to call translocations involving the IGH locus using split reads, discordant read pairs and breakpoint assembly post genome-wide alignment. ${ }^{11}$

\section{RESULTS}

Samples from 86 patients were identified (clinical parameters are summarised in online supplementary table 1). Sixty-four per cent $(55 / 86)$ of the patients were male, the median age was 62 years and the majority of patients $(81 \%, 70 / 86)$ were identified as having relapsed/refractory disease. A summary of the recurrent genomic abnormalities is shown in figure 1 . Overall the genomic landscape of the cohort was consistent with the literature published to date. Of note, the cohort was enriched for TP53 abnormalities (with sequence variants and/or copy number changes detected in $57 \%(49 / 86)$ of patients), reflecting the inclusion of a previous cohort that had been selected for $17 p$ deletion detected by fluorescence in situ hybridisation (FISH) $(\mathrm{n}=47),{ }^{7}$ as well as a high proportion of patients with relapsed/ refractory disease. Both TP53 copy number loss (18/86, 21\%) and TP53 mutation plus copy number loss $(24 / 86,28 \%)$ were more common compared with TP53 mutation alone (7/86, 8\%).

\section{Novel sequence variant findings}

A list of all sequence variants detected is shown in online supplementary table 3 . Overall, we detected 146 mutations in 31 of the 104 genes analysed and found at least one mutation in $80 \%$ of the cohort (69/86 cases, median of 2 mutations per case, range 0-6 mutations per case). Among the most frequently mutated genes were TP53 (36\%), KRAS (22.1\%), NRAS (15.1\%), FAM46C (8.1\%), DIS3 (8.1\%), TET2 (5.8\%) and FGFR3 (5.8\%). Despite this, specific variants that have not previously been described in myeloma were detected. As expected, KRAS mutations involved canonical hot spot codons Gln61 and Gly12/13. We also detected an Ile24Asn and Tyr64Asp, and mutations involving Ala59 and Lys117 each in two cases, which are only rarely described in larger cohorts (CoMMpass Interim Analysis 12 (IA12) data set). In addition, we detected novel mutations in RASA2 and PTPN11 previously undescribed in myeloma. Collectively, the incidence of RAS/MAPK pathway sequence variants (in KRAS, NRAS, BRAF, RASA2 and PTPN11) was 46.5\% (40 of 86 patients).

Three CRBN mutations (Thr361Cysfs*7, Gly151*, Pro382Arg) were detected in two patients, and one of these patients had relapsed/refractory disease and had been extensively treated with immunomodulatory drugs (IMiDs). Cereblon (encoded by $C R B N$ ) is important in mediating the anti-myeloma activity of the IMiD class of therapeutics, and therefore the pattern of mutations seen in CRBN is truncation or deleterious missense mutation in critical functional domains. ${ }^{12}$ The missense mutation we detected (Pro382Arg) has not been previously described in myeloma but is in the IMiD-binding domain next to a previously described missense mutation. ${ }^{13}$ No mutations in other genes associated with IMiD resistance (IKZF1, IKZF3 or IRF4) were identified.

\section{Novel copy number changes}

A diverse range of recurrent gains and losses were detected in the cohort (figure 2), including numerous novel focal copy number changes (table 1 and online supplementary table 4).

We observed previously described rare biallelic losses in tumour suppressors BIRC2/BIRC3, CDKN2A, PTPRD, FAF1/ $C D K N 2 C$ and $R B 1^{31415}$ consistent with the relapsed/refractory nature of the cohort, as well as novel biallelic losses in VAV3 and MYH4. Two of the BIRC2/BIRC3 biallelic losses occurred in patients with $\mathrm{t}(4 ; 14)$, an association that has been previously noted. ${ }^{16}$ In addition, we observed that the biallelic loss was intragenic for MYH4 (exons 23-39), the three RB1 deletions (exons 5-17, exons 18-23, exons 8-11) and PTPRD (exons 15-26).

\section{Novel structural variants}

Fifty per cent $(43 / 86)$ of patients had a structural variant (SV) involving the IGH locus detected by sequencing, which are listed in online supplementary table 5. These SVs included those with breakpoints within the vicinity of genes known to be involved in chromosomal translocations in myeloma, including CCND1 (18 cases), FGFR3/WHSC1 (10 cases), MAF (3 cases), CCND3 ( 2 cases), CCND2 (1 case) and MAFB (1 case). Two cases also harboured a SV involving MYC. Multiple SVs were detected involving the IGH locus and non-canonical candidate partner genes (selected based on proximity to the translocation breakpoint), and these are listed in table 2 .

\section{DISCUSSION}

Genomic characterisation in multiple myeloma is becoming increasingly adopted in the diagnostic laboratory in order to enhance diagnosis, prognosis and choice of therapy. Despite the genomically well-characterised nature of multiple myeloma, review of clinical sequencing data from our diagnostic laboratory has detected multiple novel genomic findings, including novel sequence variants, copy number changes and SVs involving the IGH locus.

Dysregulation of the RAS/MAPK pathway is an established mechanism in malignant plasma cells. While this is typically achieved with activating NRAS, KRAS and BRAF mutations, we have described mutations in RASA2 not previously described in myeloma. Interestingly, despite the general effect of mutations in RASA2 being loss of function (encoding for a GTPase-activating protein that promotes the conversion of active RAS to an inactive state, thus negatively 


\section{|GH translocation ||||||||| ||||||||||||||||||||||||||||||||||||||||||||||||||||||||||||}

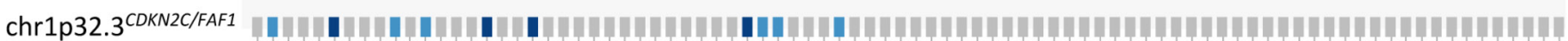

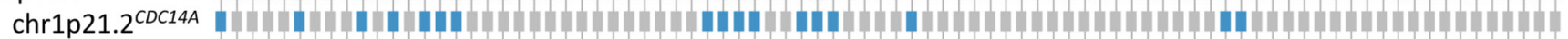
chr1p13.3 AHCYL1 H chr1p12 FAMa6c

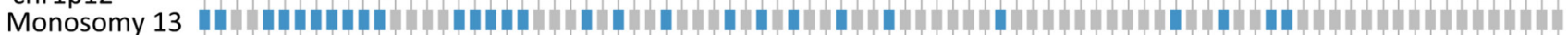
Novel loss

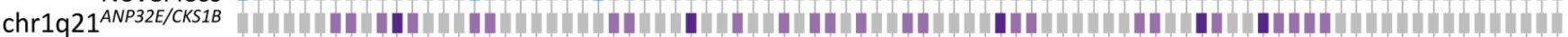

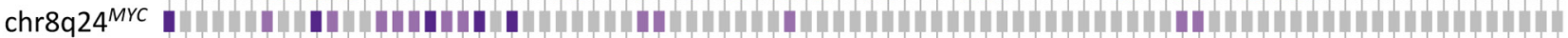

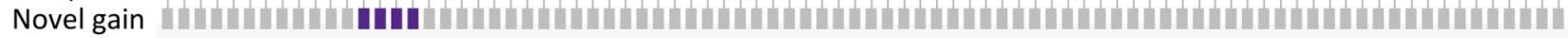

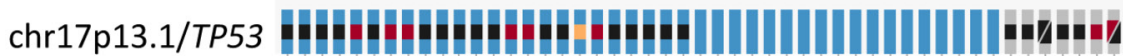

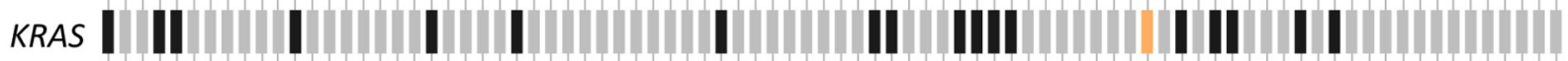

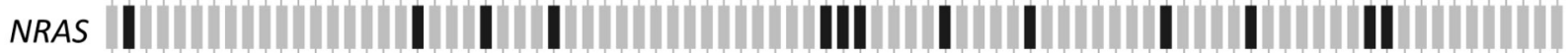

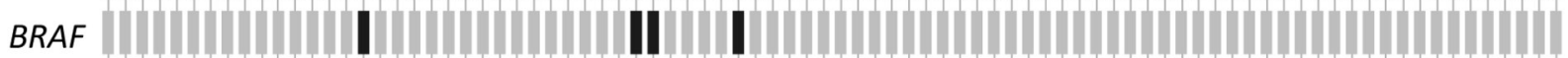
rass2 ||||||||||||||||||||||||||||||||||||||||||||||||||||||||||||||||||||||||||||||||||||||| RB1 |||||||||||||||||||||||||||||||||||||||||||||| ||||||||||||||||||||||||||||||||||||||||||||-

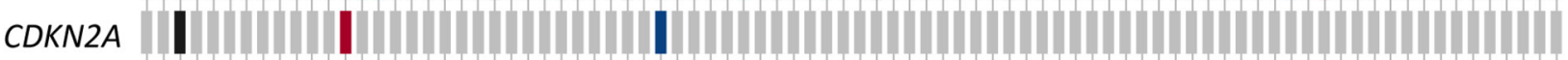

ATM

BIRC3 ||||||||||||

DIS3 ||||||| || ||||||||||||

EP300

FAM46C

FGFR3 ||I||||||

HIST1H1E

LTB

TET2

TRAF3

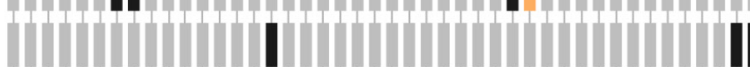

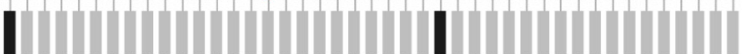
||||||||||||||

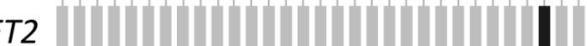
IIIIIIIII

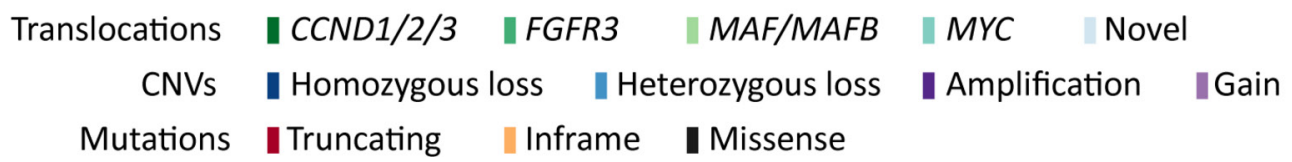

Figure 1 Genomic alterations in 86 multiple myelomas detected by targeted sequencing. Each column represents an individual sample and each row represents a gene or genomic location. Key copy number changes including loss of chromosomes 1 p, 13 and 17p, and gain of chromosomes 1q and $8 q$, are indicated, as well as novel gains and losses detected by this study. Recurring mutations (mutated in at least three cases) and structural variants involving the IGH locus are also shown. A complete list of mutations and IGH translocations are found in online supplementary tables 3-5. CNV, copy number variation; IGH, immunoglobulin heavy chain.

regulating this pathway), we detected two missense mutations occurring in the same codon (Arg511Cys and Arg511His). The COSMIC database (http://cancer.sanger.ac.uk/cosmic) indicates this codon to be the most frequently mutated codon in RASA2 (however with only six cases reported in skin, gastric and oesophageal cancers). Therefore our data provide further evidence for this codon as a recurrently mutated hot spot in RASA2 and that RASA2 suppression provides an alternative mechanism for the constitutive activation of RAS signalling in myeloma. Likewise, we also detected activating mutations in PTPN11 (Asp61His and Ala72Val), an oncogene encoding for the SHP2 protein leading to increased RAS/ MAPK pathway signalling conventionally associated with the autosomal disorder Noonan syndrome as well as juvenile myelomonocytic leukaemia. ${ }^{17}$

From a clinical management perspective, we make the important observation that of patients with TP53 abnormalities,
8\% had TP53 mutations only without copy number change. Despite both deletion of $17 \mathrm{p}$ detected by FISH and TP53 mutations being associated with inferior outcomes in myeloma, ${ }^{7} \mathrm{FISH}$ for $17 \mathrm{p}$ deletion alone is most commonly performed as part of routine prognostic investigation. Importantly, our analysis of patients in the CoMMpass data set with deletion of $17 \mathrm{p}$ and those with TP53 mutation only (ie, without deletion of $17 \mathrm{p}$ ) demonstrated no statistically significant difference in progression-free or overall survival. Therefore, in order to identify all patients with abrogated p53 function (and thus those at risk of inferior outcomes and potentially requiring novel treatment approaches), both mutations and copy number changes need to be assessed.

In terms of copy number changes, we focused on high-level focal amplifications and biallelic deletions due to their ability to give insight into potentially important oncogenic and tumour 


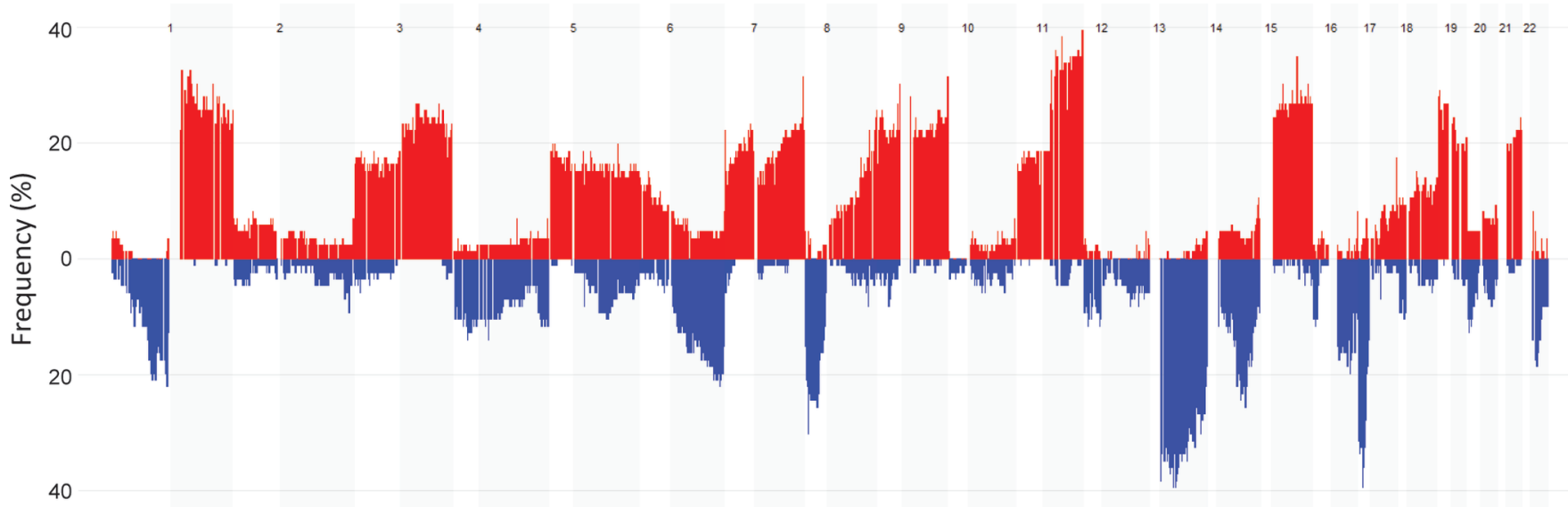

Figure 2 Genome-wide frequency plot of copy number gains (red) and losses (blue) in 86 multiple myelomas.

suppressor pathways. A high level focal copy number gain was detected on chromosome $7 \mathrm{p}$ involving IL6 (as well as numerous other genes; see online supplementary table 4). The IL6-IL6R axis is important in multiple myeloma pathogenesis, ${ }^{18}$ and IL6R is part of the typical $1 \mathrm{q}$ gain seen in multiple myeloma and has been previously implicated in high-level amplifications. ${ }^{19}$ Lower level gains are described in the CoMMpass IA12 data set and are associated with increased IL6 expression by RNA sequencing.

A focal amplification was detected involving 17p11.2 in three patients. This copy number amplification has not been specifically described in the literature to date; however, one patient in the CoMMpass IA12 data set is documented as having high-level copy number gain at the same locus. While this amplification involves many genes, one gene of particular interest inside the common amplified region is TNFRSF13B, which encodes TACI, the receptor for BAFF/APRIL which has been shown to be variably expressed in myeloma and is associated with plasma cell differentiation. ${ }^{20}$ RNA sequencing data from the CoMMpass IA12 data set demonstrate that the patient with amplification of a similar region had increased expression of TNFRSF13B relative to the rest of the cohort. Interestingly, both $7 \mathrm{p} 15.3$ and $17 \mathrm{p} 11.2$ have also been shown to contain germline variants associated with a genetic susceptibility to myeloma. ${ }^{21} 22$ In addition to focal copy number amplifications, our observation of partial deletion of prognostic markers such as $R B 1$ is important as these are potentially below the resolution of FISH and require sequencing approaches to detect.

In addition to recurrent IGH SVs, multiple SVs were detected involving the IGH locus and non-canonical candidate partner genes (selected based on proximity to the translocation breakpoint) including the following:

- CD46, which has a role in innate immune recognition ${ }^{23}$ and has been demonstrated to have increased expression in myeloma cell lines and primary myeloma cells particularly in association with $1 \mathrm{q}$ copy number gains. ${ }^{24}$

\begin{tabular}{|c|c|c|c|}
\hline Sample & Approximate genomic location* & Copies & $\begin{array}{l}\text { Approximate } \\
\text { size }\end{array}$ \\
\hline MM16 & 7p15.3 (chr7:20015001-23940000) & 30 & $4 \mathrm{Mb}$ \\
\hline MM13 & 17p11.2 (chr17:16540001-17305000) & 27 & $765 \mathrm{~kb}$ \\
\hline MM14 & 17p11.2 (chr17:16225000-18230000) & 6 & $2 \mathrm{Mb}$ \\
\hline MM15 & 17p11.2 (chr17:16175000-18105000) & 6 & $2 \mathrm{Mb}$ \\
\hline
\end{tabular}

*Coding genes within this interval are listed in online supplementary table 4.
- TXNDC5, which has recently been described as a rare but recurrent translocation in myeloma and in this study was detected in a patient with a high hyperdiploid karyotype consistent with a previous report. ${ }^{5}$

- TRAP1, which is a member of the Hsp90 family that functions as an antiapoptotic protein by controlling ubiquitination of several mitochondrial proteins. ${ }^{25}$

- ZBTB38, which is a transcriptional repressor that regulates DNA replication with overexpression expected to have the same functional effect as loss of the tumour suppressor gene $R B B P 6$ (ie, increase DNA damage at common fragile sites). ${ }^{26}$

- $R C C 2$, which is involved in cell cycle regulation. ${ }^{27}$

- BCL7A, which is expressed throughout the $\mathrm{B}$ cell lineage but tends to be downregulated in normal plasma cells. ${ }^{28}$

Coding and non-coding mutations have been reported in $B C L 7 A$ previously in myeloma. ${ }^{129}$ Despite $B C L 7 A$ being initially described as a putative tumour suppressor in other B cell malignancies, the spectrum of genomic lesions seen in BCL7A to date from the literature, the CoMMpass IA12 data set (non-coding mutations, relatively high proportion of inframe changes and loss of start codons, absence of nonsense/frameshift mutations and copy number losses) and our finding of BCL7A as a potential immunoglobulin fusion partner is atypical for classical tumour suppressor biology, suggesting further study is warranted to clarify its potential biological role in myeloma.

Despite extensive genomic characterisation of patients with myeloma in the literature and the public availability of more than 1000 exomes and RNA sequencing data, we have demonstrated that there are still novel genomic findings to detect even in routine diagnostic sequencing. Findings such as ours contribute to the overall documentation of the genomic landscape of myeloma and provide insights into plasma cell pathobiology,

\begin{tabular}{|c|c|c|}
\hline Sample & $\begin{array}{l}\text { Candidate partner } \\
\text { gene }\end{array}$ & Description \\
\hline MM44 & $R C C 2$ & $\begin{array}{l}\text { Previously undescribed; RNA-sequencing } \\
\text { confirmed } R C C 2 \text { overexpression }\end{array}$ \\
\hline MM33 & TRAP1 & Previously undescribed \\
\hline MM34 & ZBTB38 & Described in CoMMpass Interim Analysis 12 \\
\hline MM35 & $C D 46$ & Previously undescribed \\
\hline MM13 & GABRG1/GNPDA2 & Previously undescribed \\
\hline MM64 & TXNDC5 & Rare previously described translocation ${ }^{5}$ \\
\hline MM12 & $B C L 7 A$ & Previously undescribed \\
\hline
\end{tabular}

Ryland GL, et al. J Clin Pathol 2018;71:895-899. doi:10.1136/jclinpath-2018-205195 
but also illustrate the importance and utility of collaborative initiatives such as the CoMMpass study in order to centralise and aggregate genomic data with an aim to better understand the genomic landscape that underpins this disease. In addition, we have shown that comprehensive clinically relevant genomic assessment detecting a diversity of genomic lesions from simple mutations to structural variations in myeloma can be performed efficiently with a single hybridisation-based assay in the diagnostic laboratory.

\section{Take home messages}

- Comprehensive genomic characterisation of sequence variant, copy number changes and immunoglobulin heavy chain (IGH) translocation is possible in the diagnostic department with a single hybridisation, next-generation sequencing-based assay and yields novel genomic findings.

- Sequence variant detection in myeloma is important in order to completely capture TP53 abnormalities and those with inferior outcomes.

- Novel genomic changes giving clues to pathogenesis are still identifiable in myeloma despite its extensive published genomic characterisation.

- We have detected multiple novel candidate IGH partners including RCC2, TRAP1, CD46 and BCL7A.

\section{Handling editor Runjan Chetty.}

Contributors GLR, KJ, MD, HMP and PB contributed to study concept. GLR, KJ and $J G$ performed the experiments. GLR, KJ, JM, YK and PB analysed the data. MeC, MaC and $K Y$ contributed clinical samples and collected clinical data. GLR and PB drafted the manuscript. All authors approved the final version of the manuscript.

Funding We gratefully acknowledge funding support from The Snowdome Foundation (http://snowdome.org.au/) and Vision Super (https://www.visionsuper. com.au/)

Competing interests None declared.

Patient consent Not required.

Ethics approval This study was performed with approval from the ethics committee of the Peter MacCallum Cancer Centre and the National Research Ethics Service Committee London.

Provenance and peer review Not commissioned; externally peer reviewed.

(c) Article author(s) (or their employer(s) unless otherwise stated in the text of the article) 2018. All rights reserved. No commercial use is permitted unless otherwise expressly granted.

\section{REFERENCES}

1 Chapman MA, Lawrence MS, Keats JJ, et al. Initial genome sequencing and analysis of multiple myeloma. Nature 2011;471:467-72.

2 Bolli N, Avet-Loiseau H, Wedge DC, et al. Heterogeneity of genomic evolution and mutational profiles in multiple myeloma. Nat Commun 2014;5:2997.

3 Lohr JG, Stojanov P, Carter SL, et al. Widespread genetic heterogeneity in multiple myeloma: implications for targeted therapy. Cancer Cell 2014;25:91-101.

4 Walker BA, Boyle EM, Wardell CP, et al. Mutational Spectrum, Copy Number Changes, and Outcome: Results of a Sequencing Study of Patients With Newly Diagnosed Myeloma. J Clin Oncol 2015;33:3911-20.

5 Cleynen A, Szalat R, Kemal Samur M, et al. Expressed fusion gene landscape and its impact in multiple myeloma. Nat Commun 2017;8:1893.
6 Blombery PA, Ryland GL, Markham J, et al. Detection of clinically relevant early genomic lesions in B-cell malignancies from circulating tumour DNA using a single hybridisation-based next generation sequencing assay. Br $\mathrm{J}$ Haematol. Published Online First: 7 Sep 2017. doi:10.1111/bjh.14919.

7 Chin M, Sive Jl, Allen C, et al. Prevalence and timing of TP53 mutations in del(17p) myeloma and effect on survival. Blood Cancer J 2017;7:e610.

8 Walker BA, Wardell CP, Johnson DC, et al. Characterization of IGH locus breakpoints in multiple myeloma indicates a subset of translocations appear to occur in pregerminal center B cells. Blood 2013;121:3413-9.

9 Li H, Durbin R. Fast and accurate short read alignment with Burrows-Wheeler transform. Bioinformatics 2009;25:1754-60.

10 McKenna A, Hanna M, Banks E, et al. The Genome Analysis Toolkit: a MapReduce framework for analyzing next-generation DNA sequencing data. Genome Res 2010;20:1297-303.

11 Cameron DL, Schröder J, Penington JS, et al. GRIDSS: sensitive and specific genomic rearrangement detection using positional de Bruijn graph assembly. Genome Res 2017:27:2050-60.

12 Kortüm KM, Zhu YX, Shi CX, et al. Cereblon binding molecules in multiple myeloma. Blood Rev 2015:29:329-34

13 Kortüm KM, Mai EK, Hanafiah NH, et al. Targeted sequencing of refractory myeloma reveals a high incidence of mutations in CRBN and Ras pathway genes. Blood 2016;128:1226-33.

14 Chavan SS, He J, Tytarenko R, et al. Bi-allelic inactivation is more prevalent at relapse in multiple myeloma, identifying RB1 as an independent prognostic marker. Blood Cancer J 2017;7:e535.

15 Kamada Y, Sakata-Yanagimoto M, Sanada M, et al. Identification of unbalanced genome copy number abnormalities in patients with multiple myeloma by single-nucleotide polymorphism genotyping microarray analysis. Int J Hematol 2012;96:492-500.

16 Shah V, Sherborne AL, Walker BA, et al. Prediction of outcome in newly diagnosed myeloma: a meta-analysis of the molecular profiles of 1905 trial patients. Leukemia 2018:32:102-10.

17 Caye A, Strullu M, Guidez F, et al. Juvenile myelomonocytic leukemia displays mutations in components of the RAS pathway and the PRC2 network. Nat Genet 2015;47:1334-40

18 French JD, Tschumper RC, Jelinek DF. Analysis of IL-6-mediated growth control of myeloma cells using a gp130 chimeric receptor approach. Leukemia 2002;16:1189-96.

19 Kim SY, Min HJ, Park HK, et al. Increased copy number of the interleukin-6 receptor gene is associated with adverse survival in multiple myeloma patients treated with autologous stem cell transplantation. Biol Blood Marrow Transplant 2011:17:810-20.

20 Moreaux J, Cremer FW, Reme T, et al. The level of TACl gene expression in myeloma cells is associated with a signature of microenvironment dependence versus a plasmablastic signature. Blood 2005;106:1021-30.

21 Chubb D, Weinhold N, Broderick P, et al. Common variation at 3q26.2, 6p21.33, 17p11.2 and 22q13.1 influences multiple myeloma risk. Nat Genet 2013;45:1221-5.

22 Li N, Johnson DC, Weinhold N, et al. Multiple myeloma risk variant at 7p15.3 creates an IRF4-binding site and interferes with CDCA7L expression. Nat Commun 2016;7:13656

23 Elward K, Griffiths M, Mizuno M, et al. CD46 plays a key role in tailoring innate immune recognition of apoptotic and necrotic cells. J Biol Chem 2005;280:36342-54.

24 Sherbenou DW, Aftab BT, Su Y, et al. Antibody-drug conjugate targeting CD46 eliminates multiple myeloma cells. J Clin Invest 2016;126:4640-53.

25 Amoroso MR, Matassa DS, Laudiero G, et al. TRAP1 and the proteasome regulatory particle TBP7/Rpt3 interact in the endoplasmic reticulum and control cellular ubiquitination of specific mitochondrial proteins. Cell Death Differ 2012:19:592-604.

26 Miotto B, Chibi M, Xie P, et al. The RBBP6/ZBTB38/MCM10 axis regulates DNA replication and common fragile site stability. Cell Rep 2014;7:575-87.

27 Bruun J, Kolberg M, Ahlquist TC, et al. Regulator of Chromosome Condensation 2 Identifies High-Risk Patients within Both Major Phenotypes of Colorectal Cancer. Clin Cancer Res 2015:21:3759-70.

28 Ramos-Medina R, Montes-Moreno S, Maestre L, et al. BCL7A protein expression in normal and malignant lymphoid tissues. Br 」 Haematol 2013:160:106-9.

29 Manojlovic Z, Christofferson A, Liang WS, et al. Comprehensive molecular profiling of 718 Multiple Myelomas reveals significant differences in mutation frequencies between African and European descent cases. PLoS Genet 2017;13:e1007087. 